\title{
Noncoding RNAs in Cardiovascular Disease
}

\author{
Masaharu Kataoka and Da-Zhi Wang
}

Abstract

For decades, it has been recognized that proteins, which are encoded by our genomes via transcription and translation, are building blocks that play vital roles in almost all biological processes. Mutations identified in many proteincoding genes are linked to various human diseases. However, this "proteincentered" dogma has been challenged in recent years with the discovery that majority of our genome is "noncoding" yet transcribed. Noncoding RNA has become the focus of "next generation" biology. Here, we review the emerging field of noncoding RNAs, including microRNAs (miRNAs) and long noncoding RNAs (lncRNAs), and their function in cardiovascular biology and disease.

\section{Keywords}

Cardiac disease $\bullet$ Heart development $\bullet$ Long noncoding RNAs $\bullet$ MicroRNAs

\subsection{Introduction}

When the human genome project was completed, it was surprising that only about 20,000-25,000 protein-coding genes exist in our species, with less than $2 \%$ of the human genome used for coding proteins. What are the functions of noncoding sequences, which make up more than $98 \%$ of our genome? We are now finding answers with the recognition that the majority of the genome is actively transcribed to produce thousands of noncoding transcripts, including microRNAs (miRNAs) and long noncoding RNAs (lncRNAs), in many cell types and tissues. miRNAs are a class of small noncoding RNAs ( 22 nucleotides) and were first discovered in

M. Kataoka, M.D., Ph.D. • D.-Z. Wang, Ph.D. ( $\bowtie)$

Department of Cardiology, Harvard Medical School, Boston Children's Hospital, 320 Longwood Ave, Boston, MA, USA

e-mail: dwang@enders.tch.harvard.edu

T. Nakanishi et al. (eds.), Etiology and Morphogenesis of Congenital Heart Disease, DOI 10.1007/978-4-431-54628-3_44 
C. elegans two decades ago. More than 2,000 miRNAs have been found in humans, and many of them are evolutionarily conserved. By imperfect base pairing with mRNAs in a sequence-dependent manner, miRNAs repress gene expression by degrading target mRNAs and/or inhibiting their translation. Roles for miRNAs have been demonstrated in the regulation of a broad range of biological activities and diseases [1]. More recently, thousands of lncRNAs, which are transcribed noncoding RNAs greater than 200 nucleotides, were discovered and implicated in a variety of biological processes [2]. Clearly, investigating and understanding of how miRNAs and lncRNAs regulate gene expression during cardiovascular development and function will greatly facilitate therapeutic treatment of cardiovascular disease.

\section{2 miRNAs in Cardiac Development}

Global disruption of all miRNA expression in the heart is the first step to understanding the function of miRNAs in cardiac development and physiology. Dicer, a RNase III endoribonuclease, is a critical enzyme for the maturation of most miRNAs. Conventional deletion of Dicer causes early embryonic lethality in mice [3]. Disrupting miRNA expression in the early embryonic stage using Nkx2.5-Cre leads to improperly compacted ventricular myocardium in mutant embryos [4], and $\alpha$-MHC-Cre-mediated conditional deletion of Dicer causes postnatal lethality due to dilated cardiomyopathy and heart failure [5]. These studies suggest that many miRNAs have crucial roles in cardiac development. miR-1 is tissue-specifically expressed in the heart and skeletal muscle, and genetic deletion of both miR-1-1 and miR-1-2 indicated that miR-1 is required for cardiomorphogenesis and the expression of many cardiac contractile proteins [6].

\subsection{Cardiac Regeneration, Remodeling, and Ischemia Regulated by miRNAs}

Mammalian adult cardiomyocytes are terminally differentiated cells with very limited regenerative ability. A recent report identified about 40 miRNAs that strongly increased cell proliferation in neonatal mouse and rat cardiomyocytes. Two of these miRNAs, miR-590 and miR-199a, were further demonstrated to induce cardiomyocyte proliferation both in vitro and in vivo [7]. Using both gainand loss-of-function approaches in transgenic and knockout mice models, we demonstrated that the miR-17-92 cluster is required for and sufficient to induce cardiomyocyte proliferation. More specifically, we identified miR-19a/b as the major contributors among the miR-17-92 cluster to the regulation of the cardiomyocyte proliferation [8]. These studies suggest that miRNAs are key regulators of cardiomyocyte proliferation and heart regeneration, suggesting their significant therapeutic potential to treat cardiac-degeneration-associated heart disease. 
Cardiac remodeling, which is defined as an alteration in the structure (dimensions, mass, shape) of the heart, is one of the major responses of the heart to biomechanical stress and pathological stimuli. Numerous studies have demonstrated the functional involvement of many miRNAs during cardiac remodeling [9]. Recently, we and others demonstrated that miR-22, a miRNA enriched in cardiomyocytes but only mildly upregulated during cardiac hypertrophy, significantly promotes cardiac hypertrophy in vitro and in vivo [10, 11].

Ischemia is an independent risk factor of cardiovascular events, which leads to myocardial infarction (MI) and ischemia-reperfusion (I/R) injury. Several miRNAs participate in the regulation of these pathologic processes, especially cardiomyocyte apoptosis following MI and I/R injury. miR-92a, a member of the miR-17-92 cluster involved in cardiomyocyte proliferation, also participates in the control of cardiomyocyte survival by targeting integrin subunit $\alpha 5$ and eNOS. Inhibition of miR-92a by antagomir has improved cardiac function and reduced cardiomyocyte apoptosis after MI in mice [12]. miR-21 serves as an anti-apoptotic factor in MI animal models by targeting PDCD4 and repressing its expression. Interestingly, miR-21 seems to target cardiac fibroblasts, not cardiomyocytes, in the heart [13]. Conversely, miR-320 is downregulated after I/R injury. Gain- and lossof-function studies demonstrated that miR-320 promotes cardiomyocyte apoptosis via maintaining HSP20 levels [14]. Together, these studies establish miRNAs as key regulators of cardiomyocyte survival and cardiac remodeling in response to pathophysiological stresses.

\subsection{LncRNAs in Cardiac Development}

While many lncRNAs have recently been discovered, relatively little is known about their function. A novel lncRNA, Braveheart, has been defined as a critical regulator of cardiovascular commitment from embryonic stem cells (ESCs) [15]. Braveheart activates a cardiovascular gene network and functions upstream of mesoderm posterior 1, a master regulator of a common multipotent cardiovascular progenitor. Braveheart mediates the epigenetic regulation of cardiac commitment by interacting with SUZ12, a component of the polycomb repressive complex 2 (PRC2). Braveheart therefore represents the first lncRNA that defines cardiac cell fate and lineage specificity, linking lncRNAs to cardiac development and disease. It remains to be seen if Braveheart is required for normal heart development in vivo. Equally critically, it will be important to determine whether genetic mutation of the Braveheart gene is linked to human cardiovascular disorders. Nevertheless, the discovery of Braveheart will significantly impact the cardiovascular research field and link lncRNAs to human cardiovascular disease.

Fendrr, another novel IncRNA, has been defined as an essential regulator of heart and body wall development. Fendrr is expressed in the mouse lateral plate mesoderm, from which precursors for the heart and body wall are derived, and the knockout of Fendrr resulted in defects in heart development [16]. Like Braveheart, Fendrr interacts with the PRC2 complex to regulate gene expression. It is expected 
that many more lncRNAs will be found to play important roles in cardiovascular development and function.

\subsection{Noncoding RNAs in Cardiac Disease}

The expression and function of multiple miRNAs have been associated with human cardiovascular disease. Recent studies also linked several lncRNAs to heart disease. ANRIL, a lncRNA, was identified as a risk factor for coronary disease [17]. Though it is still not fully understood how ANRIL functions, evidence suggests that this lncRNA may participate in the regulation of histone methylation [18]. Another lncRNA MIAT (myocardial infarction-associated transcript) (or Gomafu/RNCR2) was identified as a risk factor associated with patients with myocardial infarction [19]. However, how MIAT controls MI status remains largely unknown. Intriguingly, the genetic loci that encode MYH6 and MYH7, the main myosin heavy chain genes in cardiac muscle, appear to produce a noncoding antisense transcript (Myh7as). Myh7-as transcription may regulate the ratio of Myh6 and $M y h 7$, altering the function of muscle contraction [20].

We have just started the era of "noncoding." We are looking forward to see more and more reports on the roles of noncoding RNAs in the regulation of a variety of essential biological processes. Furthermore, with efficient strategies for gain- and loss-of-function investigations, more fruitful work about the molecular mechanism and therapeutic application of noncoding RNAs in cardiovascular disease will emerge.

Acknowledgments Work in Dr. Wang's laboratory was supported by the March of Dimes Foundation and National Institutes of Health. We thank FeiFei Wang for critical reading of this commentary. Masaharu Kataoka was supported by Banyu Life Science Foundation International. DZ Wang is an established investigator of the American Heart Association.

Open Access This chapter is distributed under the terms of the Creative Commons AttributionNoncommercial 2.5 License (http://creativecommons.org/licenses/by-nc/2.5/) which permits any noncommercial use, distribution, and reproduction in any medium, provided the original author(s) and source are credited.

The images or other third party material in this chapter are included in the work's Creative Commons license, unless indicated otherwise in the credit line; if such material is not included in the work's Creative Commons license and the respective action is not permitted by statutory regulation, users will need to obtain permission from the license holder to duplicate, adapt or reproduce the material.

\section{References}

1. Espinoza-Lewis RA, Wang DZ. MicroRNAs in heart development. Curr Top Dev Biol. 2012;100:279-317. doi:10.1016/B978-0-12-387786-4.00009-9.

2. Ulitsky I, Bartel DP. lincRNAs: genomics, evolution, and mechanisms. Cell. 2013;154:26-46. doi:10.1016/j.cell.2013.06.020. 
3. Bernstein E, Kim SY, Carmell MA, et al. Dicer is essential for mouse development. Nat Genet. 2003;35:215-7.

4. Zhao Y, Ransom JF, Li A, et al. Dysregulation of cardiogenesis, cardiac conduction, and cell cycle in mice lacking miRNA-1-2. Cell. 2007;129:303-17.

5. Chen JF, Murchison EP, Tang R, et al. Targeted deletion of Dicer in the heart leads to dilated cardiomyopathy and heart failure. Proc Natl Acad Sci U S A. 2008;105:2111-6. doi:10.1073/ pnas.0710228105.

6. Heidersbach A, Saxby C, Carver-Moore K, et al. MicroRNA-1 regulates sarcomere formation and suppresses smooth muscle gene expression in the mammalian heart. Elife. 2013. doi:10. 7554/eLife.01323.

7. Eulalio A, Mano M, Dal Ferro M, et al. Functional screening identifies miRNAs inducing cardiac regeneration. Nature. 2012;492:376-81. doi:10.1038/nature11739.

8. Chen J, Huang ZP, Seok HY, et al. mir-17-92 cluster is required for and sufficient to induce cardiomyocyte proliferation in postnatal and adult hearts. Circ Res. 2013;112:1557-66.

9. Small EM, Olson EN. Pervasive roles of microRNAs in cardiovascular biology. Nature. 2011;469:336-42. doi:10.1038/nature09783.

10. Huang ZP, Chen J, Seok HY, et al. MicroRNA-22 regulates cardiac hypertrophy and remodeling in response to stress. Circ Res. 2013;112:1234-43. doi:10.1161/CIRCRESAHA. 112.300658 .

11. Gurha P, Abreu-Goodger C, Wang T, et al. Targeted deletion of microRNA-22 promotes stress-induced cardiac dilation and contractile dysfunction. Circulation. 2012;125:2751-61. doi:10.1161/CIRCULATIONAHA.111.044354.

12. Bonauer A, Carmona G, Iwasaki M, et al. MicroRNA-92a controls angiogenesis and functional recovery of ischemic tissues in mice. Science. 2009;324:1710-3. doi:10.1126/science. 1174381 .

13. Dong S, Cheng Y, Yang J, et al. MicroRNA expression signature and the role of microRNA-21 in the early phase of acute myocardial infarction. J Biol Chem. 2009;284:29514-25. doi:10. 1074/jbc.M109.027896.

14. Ren XP, Wu J, Wang X, et al. MicroRNA-320 is involved in the regulation of cardiac ischemia/reperfusion injury by targeting heat-shock protein 20. Circulation. 2009;119:2357-66. doi:10.1161/CIRCULATIONAHA.108.814145.

15. Klattenhoff CA, Scheuermann JC, Surface LE, et al. Braveheart, a long noncoding RNA required for cardiovascular lineage commitment. Cell. 2013;152:570-83. doi:10.1016/j.cell. 2013.01.003.

16. Grote P, Wittler L, Hendrix D, et al. The tissue-specific lncRNA Fendrr is an essential regulator of heart and body wall development in the mouse. Dev Cell. 2013;24:206-14. doi:10.1016/j.devcel.2012.12.012.

17. Broadbent HM, Peden JF, Lorkowski S, et al. Susceptibility to coronary artery disease and diabetes is encoded by distinct, tightly linked SNPs in the ANRIL locus on chromosome 9p. Hum Mol Genet. 2008;17:806-14.

18. Yap KL, Li S, Muñoz-Cabello AM, et al. Molecular interplay of the noncoding RNA ANRIL and methylated histone $\mathrm{H} 3$ lysine 27 by polycomb $\mathrm{CBX} 7$ in transcriptional silencing of INK4a. Mol Cell. 2010;38:662-74. doi:10.1016/j.molcel.2010.03.021.

19. Ishii N, Ozaki K, Sato H, et al. Identification of a novel non-coding RNA, MIAT, that confers risk of myocardial infarction. J Hum Genet. 2006;51:1087-99.

20. Pandya K, Smithies O. $\beta$-MyHC and cardiac hypertrophy: size does matter. Circ Res. 2011;109:609-10. doi:10.1161/CIRCRESAHA.111.252619. 